Document downloaded from:

http://hdl.handle.net/10251/55340

This paper must be cited as:

Díaz Caballero, E.; Belenguer, A.; Esteban González, H.; Boria Esbert, VE. (2014). Extending the cascading by pairs of multiport generalised scattering matrices for characterizing the connected ports. IEEE Microwave and Wireless Components Letters. 24(11):733-735. doi:10.1109/LMWC.2014.2348181.

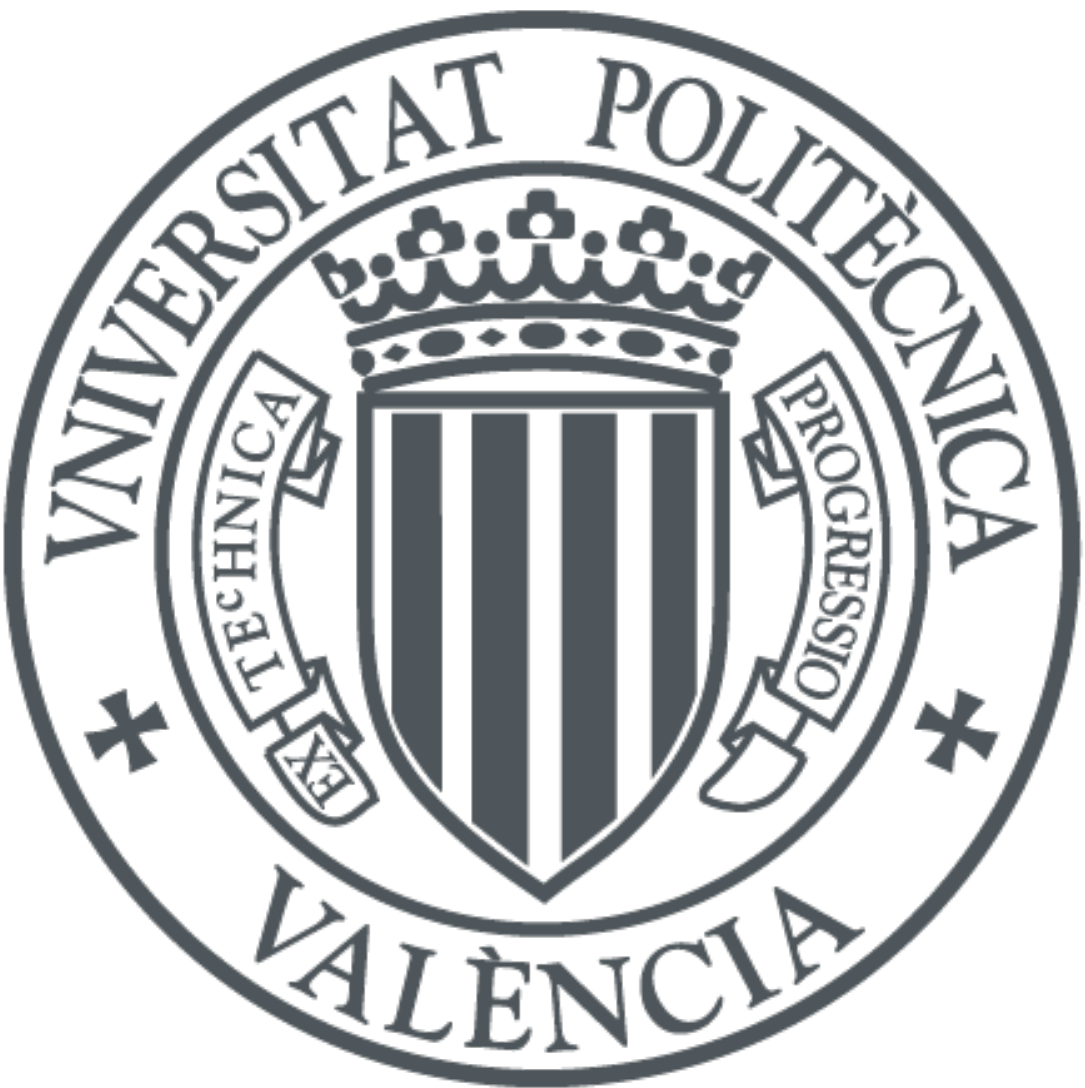

The final publication is available at

http://dx.doi.org/10.1109/LMWC.2014.2348181

Copyright Institute of Electrical and Electronics Engineers (IEEE)

Additional Information 


\title{
Extending the cascading by pairs of multiport generalized scattering matrices for characterizing the connected ports
}

\author{
Elena Diaz Caballero, Angel Belenguer, Member, IEEE, Héctor Esteban, Member, IEEE and \\ Vicente E. Boria, Senior Member, IEEE
}

\begin{abstract}
An extension to the traditional technique for cascading generalized scattering matrices (GSMs) by pairs is presented. It can solve the connection of an arbitrary number of networks, with an arbitrary number of ports, while being quite intuitive and easy to implement. It is able to compute, not only the response at the free ports of the global network -like the traditional cascading-by-pairs does-, but also the connected ports, obtaining an extended GSM (eGSM), which can be used to find the field inside the full network for an arbitrary incidence.
\end{abstract}

Index Terms-Cascade circuits, microwave devices, generalized scattering matrices, extended generalized scattering matrices.

\section{INTRODUCTION}

The cascade connection of GSMs has become a very typical example of a divide-and-conquer strategy. A complex device is divided into simple building blocks (steps, resonators, lines, etc.), then the GSM of each block is obtained using the most appropriate numerical or analytical technique and, finally, the cascade connection of all the GSMs is calculated, obtaining the GSM of the whole connected network.

Unfortunately, the internal behavior of the connected network is completely lost after performing the traditional cascading-by-pairs procedure [1], [2], so that, for instance, it is impossible to recover the fields inside the network. Therefore, an iterative Krylov's based technique was proposed in [3]. For an arbitrary external excitation, that technique was able to provide the modal weights, not only at the free ports, but also at the connected ones. However, in this work, the traditional cascading expressions of multiport and multimodal GSMs will be modified so that the resulting scattering matrix will be an extended version of a typical GSM, i.e. an extended generalized scattering matrix (eGSM). This eGSM will return the emergent modal weights at the free and connected ports for any arbitrary incidence at the free ports. This approach is more intuitive and easier to implement than the one in [3], providing with faster results when calculating the whole multimodal response of the connected network.

\section{Definition AND CASCADING OF EXTENDED GSMs}

First, we should define a matrix that can be used to compute the full modal response at every port of the connected network

E. Diaz Caballero, H. Esteban and V. E. Boria are with the Instituto de Telecomunicaciones y Aplicaciones Multimedia (iTEAM), Universitat Politecnica de Valencia, 46022 Valencia, Spain (e-mail: eldiaca@iteam.upv.es).

A. Belenguer is with the Departamento de Ingenieria Electrica, Electronica Automatica y Comunicaciones, Universidad de Castilla-La Mancha, 16071 Cuenca, Spain (e-mail: angel.belenguer@uclm.es). for an arbitrary incidence at its free ports. This extended generalized scattering matrix, $\underline{S}_{E}$, would relate the weights of the incident modes at the free ports, $\underline{a}_{F}$, and the reflected modal weights at the free, $\underline{b}_{F}$, and connected ports, $\underline{b}_{C}$, this way

$$
\underline{\underline{S}}_{E}=\left(\begin{array}{c}
\underline{\underline{S}}_{F F} \\
\underline{\underline{S}}_{C F}
\end{array}\right) \Rightarrow\left(\begin{array}{c}
\underline{b}_{F} \\
\underline{\underline{b}}_{C}
\end{array}\right)=\left(\begin{array}{c}
\underline{\underline{S}}_{F F} \\
\underline{\underline{S}}_{C F}
\end{array}\right) \underline{a}_{F}
$$

If we had a simple network containing just one single block, thus having just free ports, $\underline{\underline{S}}_{C F}$ would be a null matrix, i.e. this simple network can be fully described by a traditional GSM, which can be considered a particular case of eGSM.

Now, let us consider two general networks or devices, i.e device $m$ and device $n$, characterized by their eGSMs:

$$
\underline{\underline{S}}_{E}^{(m)}=\left(\begin{array}{c}
\underline{\underline{S}}_{O F, O F}^{(m)} \\
\underline{\underline{S}} \\
O C, O F
\end{array}\right) ; \quad \underline{\underline{S}}_{E}^{(n)}=\left(\begin{array}{c}
\underline{S}_{O F, O F}^{(n)} \\
\underline{\underline{S}}
\end{array}\right)
$$

These devices are going to be connected through some of their free ports. Therefore, the original set of free ports $(O F)$ must be separated into two subsets, so that the new connected ports $(C)$ can be distinguished from the ports which are going to remain free $(F)$. After applying this port reorganization and placing the new connected ports $(C)$ at the end of the reordered eGSMs, the eGSMs become

$$
\begin{aligned}
& \underline{\underline{S}}_{E}^{(m)}=\left(\begin{array}{cc}
\underline{\underline{S}}_{F, F}^{(m)} & \underline{\underline{S}}_{F, C}^{(m)} \\
\underline{\underline{S}}_{O C, F}^{(m)} & \underline{\underline{S}}_{O C, C}^{(m)} \\
\underline{\underline{S}}_{C, F}^{(m)} & \underline{\underline{S}}_{C, C}^{(m)}
\end{array}\right) \\
& \underline{\underline{S}}_{E}^{(n)}=\left(\begin{array}{cc}
\underline{\underline{S}}_{F, F}^{(n)} & \underline{\underline{S}}_{F, C}^{(n)} \\
\underline{\underline{S}}_{O C, F}^{(n)} & \underline{\underline{S}}_{O C, C}^{(n)} \\
\underline{\underline{S}}_{C, F}^{(n)} & \underline{\underline{S}}_{C, C}^{(n)}
\end{array}\right)
\end{aligned}
$$

Finally, if the remaining free ports $(\mathrm{F})$ are combined with the originally connected ports (OC) by defining a new subset (D), both eGSMs can be rewritten as follows,

$$
\begin{aligned}
& \underline{\underline{S}}_{E}^{(m)}=\left(\begin{array}{ll}
\underline{\underline{S}}_{D, F}^{(m)} & \underline{\underline{S}}_{D, C}^{(m)} \\
\underline{\underline{S}}_{C, F}^{(m)} & \underline{\underline{S}}_{C, C}^{(m)}
\end{array}\right) \\
& \underline{\underline{S}}_{E}^{(n)}=\left(\begin{array}{ll}
\underline{\underline{S}}_{D, F}^{(n)} & \underline{\underline{S}}_{D, C}^{(n)} \\
\underline{\underline{S}}_{C, F}^{(n)} & \underline{\underline{S}}_{C, C}^{(n)}
\end{array}\right)
\end{aligned}
$$

where

$$
\underline{\underline{S}}_{D, F}^{(m)}=\left(\begin{array}{c}
\underline{S}_{F, F}^{(m)} \\
\underline{\underline{S}}_{O C, F}^{(m)}
\end{array}\right) ; \quad \underline{\underline{S}}_{D, F}^{(n)}=\left(\begin{array}{c}
\underline{\underline{S}}_{F, F}^{(n)} \\
\underline{\underline{S}}_{O C, F}^{(n)}
\end{array}\right)
$$




$$
\underline{\underline{S}}_{D, C}^{(m)}=\left(\begin{array}{c}
\underline{S}_{F, C}^{(m)} \\
\underline{\underline{S}}_{O C, C}^{(m)}
\end{array}\right) ; \quad \underline{\underline{S}}_{D, C}^{(n)}=\left(\begin{array}{c}
\underline{\underline{S}}_{F, C}^{(n)} \\
\underline{\underline{S}}_{O C, C}^{(n)}
\end{array}\right)
$$

These eGSMs, $\underline{\underline{S}}_{E}^{(n)}$ and $\underline{\underline{S}}_{E}^{(m)}$, can be used to calculate the emergent modal weights at the complete set of ports of each original network, $D \cup C$, for an arbitrary incidence at their originally free ports, $O F \equiv F \cup C$ :

$$
\begin{aligned}
& \left(\begin{array}{l}
\underline{b}_{D}^{(n)} \\
\underline{b}_{C}^{(n)}
\end{array}\right)=\left(\begin{array}{cc}
\underline{S}_{D, F}^{(n)} & \underline{\underline{S}}_{D, C}^{(n)} \\
\underline{\underline{S}}_{C, F}^{(n)} & \underline{\underline{S}}_{C, C}^{(n)}
\end{array}\right)\left(\begin{array}{c}
\underline{a}_{F}^{(n)} \\
\underline{a}_{C}^{(n)}
\end{array}\right) \\
& \left(\begin{array}{l}
\underline{b}_{D}^{(m)} \\
\underline{b}_{C}^{(m)}
\end{array}\right)=\left(\begin{array}{ll}
\underline{\underline{S}}_{D, F}^{(m)} & \underline{\underline{S}}_{D, C}^{(m)} \\
\underline{\underline{S}}_{C, F}^{(m)} & \underline{\underline{S}}_{C, C}^{(m)}
\end{array}\right)\left(\begin{array}{l}
\underline{a}_{F}^{(m)} \\
\underline{a}_{C}^{(m)}
\end{array}\right)
\end{aligned}
$$

Then, since both devices are going to be cascaded, the following equations can be written,

$$
\begin{gathered}
\underline{b}_{D}^{(n)}=\underline{\underline{S}}_{D, F}^{(n)} \underline{a}_{F}^{(n)}+\underline{\underline{S}}_{D, C}^{(n)} \underline{a}_{C}^{(n)} \\
\underline{b}_{C}^{(n)}=\underline{\underline{S}}_{C, F}^{(n)} \underline{a}_{F}^{(n)}+\underline{\underline{S}}_{C, C}^{(n)} \underline{a}_{C}^{(n)} \\
\underline{b}_{D}^{(m)}=\underline{\underline{S}}_{D, F}^{(m)} \underline{a}_{F}^{(m)}+\underline{\underline{S}}_{D, C}^{(m)} \underline{a}_{C}^{(m)} \\
\underline{b}_{C}^{(m)}=\underline{\underline{S}}_{C, F}^{(m)} \underline{a}_{F}^{(m)}+\underline{\underline{S}}_{C, C}^{(m)} \underline{a}_{C}^{(m)} \\
\underline{a}_{C}^{(n)}=\underline{\underline{D}}_{C}^{(n)} \underline{b}_{C}^{(m)} ; \quad \underline{a}_{C}^{(m)}=\underline{\underline{D}}_{C}^{(m)} \underline{b}_{C}^{(n)}
\end{gathered}
$$

Observing (15), it is noticeable that the emergent waves from the connected ports of device $m$ are not directly equal to the incident waves to the connected ports of device $n$, and vice versa. In this case, the matrices $\underline{\underline{D}}_{C}^{(n)}$ and $\underline{\underline{D}}_{C}^{(m)}$ have been added [3], so that it is possible to consider simple mismatches at the ports like, for example, if they are connected through a piece of empty waveguide of certain length.

In order to find the eGSM of the cascade connection, first let us suppose that the connection is exclusively excited through the free ports of the device $n$, i.e. $\underline{a}_{F}^{(m)}=0$. Then, operating with (11)-(14) and defining the following auxiliary matrices in order to simplify the notation,

$$
\begin{aligned}
& \underline{\underline{S}}_{C, C}^{(n)}=\underline{\underline{S}}_{C, C}^{(n)} \underline{\underline{D}}_{C}^{(n)} \\
& \underline{\underline{\hat{S}}}_{D, C}^{(n)}=\underline{\underline{S}}_{D, C}^{(n)} \underline{\underline{D}}_{C}^{(n)} \\
& \underline{\underline{\underline{S}}}_{C, C}^{(m)}=\underline{\underline{S}}_{C, C}^{(m)} \underline{\underline{D}}_{C}^{(m)}
\end{aligned}
$$

we can find four different blocks of the eGSM of the cascaded network. In particular, we obtain the matrix $\underline{\underline{S}}_{D, F}^{(n n)}$ that can be used to compute the mode weights which emerge through the ports of the device $n$ belonging to the $D$ set, in response to an arbitrary incidence at the free ports of this same device.

$$
\underline{\underline{S}}{ }_{D, F}^{(n n)}=\underline{\underline{S}}_{D, F}^{(n)}+\underline{\underline{S}}_{D, C}^{(n)} \underline{\underline{S}}_{C, C}^{(m)} \underline{\underline{F}}_{1} \underline{\underline{S}}_{C, F}^{(n)}
$$

where,

$$
\underline{\underline{F}}_{1}=\left(\underline{\underline{I}}-\underline{\underline{\hat{S}}}_{C, C}^{(n)} \underline{\underline{S}}_{C, C}^{(m)}\right)^{-1}
$$

Operating, we also obtain the expression for $\underline{\underline{S}}_{C, F}^{(n n)}$, which can be used to obtain the modal weights which emerge through the ports of device $n$, connected with device $m$, in response to an arbitrary incidence at the free ports of device $n$,

$$
\underline{\underline{S}}_{C, F}^{(n n)}=\underline{\underline{F}}_{1} \underline{\underline{S}}_{C, F}^{(n)}
$$

as well as $\underline{S}_{D, F}^{(m n)}$, which relates the modal weights emerging through the ports of device $m$ belonging to set $D$, in response to an arbitrary incidence at the free ports of device $n$,

$$
\underline{\underline{S}}_{D, F}^{(m n)}=\underline{\underline{\hat{S}}}_{D, C}^{(m)} \underline{\underline{F}}_{1} \underline{\underline{S}}_{C, F}^{(n)}
$$

and matrix $\underline{\underline{S}}_{C, F}^{(m n)}$, which can be used to compute the emerging modal weights at the connected ports of device $m$, in response to an arbitrary incidence at the free ports of device $n$

$$
\underline{\underline{S}}_{C, F}^{(m n)}=\underline{\underline{\hat{S}}}_{C, C}^{(m)} \underline{\underline{F}} 1 \underline{\underline{S}}_{C, F}^{(n)} .
$$

In order to calculate the other blocks of the eGSM of the cascade connection, now we consider that the connected network is only excited through the free ports of device $m$, i.e. $\underline{a}_{F}^{(n)}=0$. Therefore, operating again with (11)-(14) and defining another auxiliary matrix,

$$
\underline{\underline{S}}_{D, C}^{(m)}=\underline{\underline{S}}_{D, C}^{(m)} \underline{\underline{D}}_{C}^{(m)}
$$

we can find the other four matrix blocks of the eGSM of the connection. One of them is $\underline{\underline{S}}_{D, F}^{(n m)}$,

$$
\underline{\underline{S}}_{D, F}^{(n m)}=\underline{\underline{S}}_{D, C}^{(n)} \underline{\underline{F}}_{2} \underline{\underline{S}}_{C, F}^{(m)}
$$

where

$$
\underline{\underline{F}}_{2}=\left(\underline{\underline{I}}-\underline{\underline{\hat{S}}}_{C, C}^{(m)} \underline{\underline{S}}_{C, C}^{(n)}\right)^{-1}
$$

This matrix $\underline{S}_{D, F}^{(n m)}$ can be used to compute the emergent spectrum through the ports of device $n$ belonging to set $D$, for an arbitrary incidence at the free ports of device $m$. Matrix $\underline{S}_{C, F}^{(n m)}$ is also found,

$$
\underline{\underline{S}}_{C, F}^{(n m)}=\underline{\underline{S}}_{C, C}^{(n)} \underline{\underline{F}}_{2} \underline{\underline{S}}_{C, F}^{(m)}
$$

and it can be used to compute the modes which emerge through the connected ports of device $n$ in response to an arbitrary incidence against the free ports of device $m$. Operating, we also obtain the expression for matrix $\underline{S}_{D F}^{(m m)}$, which can be used to find the emergent spectrum through the ports of device $m$ belonging to set $D$, for an arbitrary incidence at the free ports of device $m$,

$$
\underline{\underline{S}}_{D, F}^{(m m)}=\underline{\underline{S}}_{D, F}^{(m)}+\underline{\underline{\hat{S}}}_{D, C}^{(m)} \underline{\underline{\hat{S}}}_{C, C}^{(n)} \underline{\underline{F}}_{2} \underline{\underline{S}}_{C, F}^{(m)}
$$

as well as matrix $\underline{\underline{S}}_{C, F}^{(m m)}$,

$$
\underline{\underline{S}}_{C, F}^{(m m)}=\underline{\underline{F}}_{2} \underline{\underline{S}}_{C, F}^{(m)}
$$

which relates the emerging modal weights at the connected ports of device $m$, in response to an arbitrary incidence at the free ports of the same device.

Using these blocks, it is quite straightforward to obtain a first approximation of the eGSM of the cascade

$$
\underline{\underline{S}}_{E}=\left(\begin{array}{cc}
\underline{S}_{D, F}^{(n n)} & \underline{S}_{D, F}^{(n m)} \\
\underline{S}_{D, F}^{(m n)} & \underline{S}_{D, F}^{(m m)} \\
\underline{\bar{S}}_{C, F}^{(n n)} & \underline{\bar{S}}_{C, F}^{(n m)} \\
\underline{\underline{S}}_{C, F}^{(m n)} & \underline{\underline{S}}_{C, F}^{(m m)}
\end{array}\right)
$$

However, in order to obtain an eGSM compatible with the definition made in (1), we need to separate the free ports $(F)$ 
from the original connected ones $(O C)$, that is we need to split the set $D$, which contains both types of ports, and reorder the blocks of $\underline{\underline{S}}_{E}$ so that the original connected ports are placed below the new connected ones,

$$
\underline{\underline{S}}_{E}=\left(\begin{array}{cc}
\underline{\underline{S}}_{F, F}^{(n n)} & \underline{\underline{S}}_{F, F}^{(n m)} \\
\underline{\underline{S}}_{F}^{(m n)} & \underline{\underline{S}}_{F, F}^{(m m)} \\
\underline{\underline{S}}_{C, F}^{(n n)} & \underline{\underline{S}}_{C, F}^{(n m)} \\
\underline{\underline{S}}_{O C, F}^{(n n)} & \underline{\underline{S}}_{O C, F}^{(n m)} \\
\underline{S}_{C, F}^{(m n)} & \underline{\underline{S}}_{C, F}^{(m m)} \\
\underline{S}_{O C, F}^{(m n)} & \underline{\underline{S}}_{O C, F}^{(m m)}
\end{array}\right) .
$$

After this last reordering, we can group the new connected ports $(C)$ with the original connected ones $(O C)$ to obtain a new set $(N C)$, so that the final expression for the extended GSM of the connected network is

$$
\underline{\underline{S}}_{E}=\left(\begin{array}{cc}
\underline{\underline{S}}_{F, F}^{(n n)} & \underline{\bar{S}}_{F, F}^{(n m)} \\
\underline{S}_{F, F}^{(m n)} & \underline{\bar{S}}_{F, F}^{(m m)} \\
\bar{S}_{N n)}^{(n n)} & \underline{\underline{S}}_{N C, F}^{(n m)} \\
\underline{\bar{S}}_{N n)}^{(m n)} & \underline{\underline{S}}_{N C, F}^{(m m)}
\end{array}\right)=\left(\begin{array}{c}
\underline{S}_{F, F} \\
\underline{\underline{S}}_{N C, F}
\end{array}\right)
$$

which is compatible with the definition of eGSM made in (1). Finally, if $N$ devices are connected, all of them having the same number of ports, $A$, and considering $N_{\bmod }$ modes at each port, the computational cost of the whole connection can be deduced to be $\approx N\left(4 A^{2}+4 A+4\right) N_{\text {mod }}^{3}$.

\section{RESULTS}

In this section, the extension of the cascading by pairs of generalized scattering matrices for characterizing the connected ports in a network has been tested. It has been applied for solving a designed bandpass rectangular waveguide filter whose layout and dimensions are shown in Fig. 1.

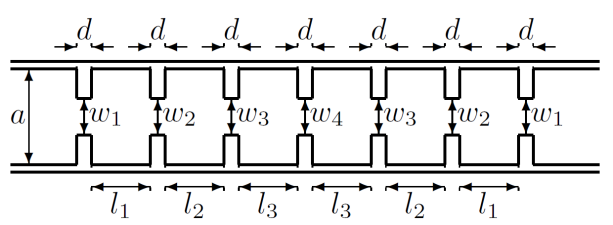

Fig. 1. Layout of the bandpass filter: $a=19.05 \mathrm{~mm}, d=1 \mathrm{~mm}$ $w_{1}=11.803 \mathrm{~mm}, w_{2}=8.620 \mathrm{~mm}, w_{3}=7.647 \mathrm{~mm}, w_{4}=7.458 \mathrm{~mm}$, $l_{1}=12.777 \mathrm{~mm}, l_{2}=14.859 \mathrm{~mm}, l_{3}=15.405 \mathrm{~mm}$.

In order to perform the analysis of the filter, first the GSM of each building block has been obtained (analytically for waveguide sections and using mode-matching [4] for steps) and then the whole connection has been performed with the new extended cascading-by-pairs technique and also with the Krylov's iterative technique in [3], obtaining the complete eGSM of the filter. Considering 20 modes at each port and analyzing 301 frequency points, $8.21 \mathrm{~s}$ have been necessary to obtain the GSMs of all the building blocks, and Table I shows the computational times for the whole connection of the network. It can be observed that while for the iterative technique it was necessary to compile the cascading routine in $\mathrm{C}++$ in order to obtain more competitive times (still worse than the ones with the new technique), the new technique is not so complex and it just deals with matrices, so an implementation in Matlab is as efficient as a compiled $\mathrm{C}++$ routine would be.

TABLE I

COMPUTATIONAL TIMES FOR CASCADING THE FILTER BLOCKS.

\begin{tabular}{|l|c|c|c|c|}
\cline { 2 - 5 } \multicolumn{1}{c|}{} & \multicolumn{2}{c|}{ New } & \multicolumn{2}{c|}{$[3]$} \\
\cline { 2 - 5 } \multicolumn{1}{c|}{} & Matlab & $\mathrm{C}++$ & Matlab & $\mathrm{C}++$ \\
\hline Total time & $5.36 \mathrm{~s}$ & $5.17 \mathrm{~s}$ & $29.23 \mathrm{~s}$ & $6.59 \mathrm{~s}$ \\
\hline Time/mode/port/freq. point & $29.68 \mu \mathrm{s}$ & $28.63 \mu \mathrm{s}$ & $161.85 \mu \mathrm{s}$ & $36.48 \mu \mathrm{s}$ \\
\hline
\end{tabular}

Figure 2 shows the global S-parameters, validating the accuracy of the new technique. And finally, Fig. 3 represents the electric field magnitude at the central bandpass frequency, which has been obtained from the modal weights at the connected ports provided by the calculated eGSM.

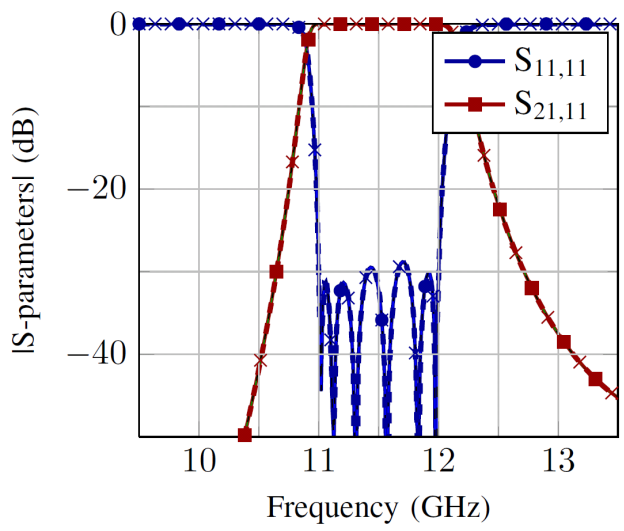

Fig. 2. Comparison of the S-parameters of the bandpass filter obtained with the new extended cascading-by-pairs technique (solid line), with the iterative cascading technique in [3] (cross marks) and with FEST3D [5] (dashed).

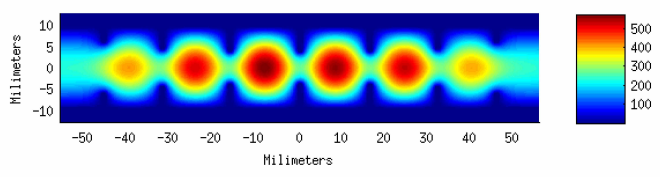

Fig. 3. Electric field magnitude inside the filter at $11.5 \mathrm{GHz}$.

\section{CONCLUSIONS}

An extension of the traditional cascading-by-pairs technique has been presented. This technique provides an extended GSM for the connection of an arbitrary number of devices with an arbitrary number of ports, so that it characterizes the response of the connected network not only at the free ports but also at the connected ones. Moreover, this technique has been tested with an example, proving to be very accurate and efficient.

\section{REFERENCES}

[1] D. M. Pozar, Microwave Engineering. John Wiley \& Sons, 2005.

[2] D. Budimir, Generalized filter design by computer optimization, 1998.

[3] A. Belenguer, E. Diaz Caballero, H. Esteban, A. L. Borja, and J. Cascon, "Krylov's Solver Based Technique for the Cascade Connection of Multiple N-Port Multimodal Scattering Matrices," Microwave Theory and Techniques, IEEE Trans. on, vol. 61, no. 2, pp. 720 -726, feb. 2013.

[4] J. Reiter and F. Arndt, "Rigorous analysis of arbitrarily shaped H- and Eplane discontinuities in rectangular waveguides by a full-wave boundary contour mode-matching method," IEEE Trans. Microwave Theory Tech., vol. 43, no. 4, pp. 796-801, April 1995.

[5] Aurora Software and Testing, http://www.fest3d.com. 